\title{
Cost-effective surveillance of an invasive species: application of info-gap theory to the Asian House Gecko Hemidactylus frenatus
}

Yang Liu ( $\sim$ Yang.Liu@murdoch.edu.au )

Qingdao Agricultural University

Penghao Wang

Food Futures Institute, Murdoch University

Melissa L. Thomas

Harry Butler Institute, Murdoch University

Dan Zheng

Qingdao Agricultural University

Simon J. McKirdy

Harry Butler Institute, Murdoch University

\section{Research Article}

Keywords: IGDT, Hemidactylus frenatus, reward function, trigger detection, native species

Posted Date: August 12th, 2021

DOI: https://doi.org/10.21203/rs.3.rs-794258/v1

License: (9) This work is licensed under a Creative Commons Attribution 4.0 International License. Read Full License 
4 Yang Liu ${ }^{1,2 *}$, Penghao Wang ${ }^{2,3}$, Melissa L. Thomas ${ }^{2}$, Dan Zheng ${ }^{1 *}$ and Simon J. McKirdy ${ }^{2 *}$

$5 \quad$ Q Qingdao Agricultural University, Shandong 266109, P.R. China

$6 \quad{ }^{2}$ Harry Butler Institute, Murdoch University, Perth, WA 6150, Australia

$7 \quad{ }^{3}$ Food Futures Institute, Murdoch University, Perth, WA 6150, Australia

8 *Corresponding author

9 E-mail:YYang.Liu@murdoch.edu.au; zhengdan1818@163.com; s.mckirdy@murdoch.edu.au 


\section{Abstract}

19 Invasive species can lead to community-level damage to the invaded ecosystem and extinction of

20 native species. Most surveillance systems for the detection of invasive species are developed

21 based on expert assessment, inherently coming with a level of uncertainty. In this research, info-

22 gap decision theory (IGDT) is applied to model and manage such uncertainty; surveillance of the

23 Asian House Gecko, Hemidactylus frenatus Duméril and Bibron, 1836 on Barrow Island, is used

24 as a case study. Our research provides a novel method for applying IGDT to determine the robust

25 population threshold $(K)$ to trigger detection, robust-optimizing surveillance costs rather than

26 minimizing surveillance costs. We demonstrate that increasing the population threshold for

27 detection increases both robustness to the errors in the model parameter estimates, and

28 opportuneness to lower surveillance costs than the accepted maximum budget. This paper

29 provides guidance for decision makers to balance robustness and required surveillance

30 expenditure. IGDT offers a novel method to model and manage the uncertainty prevalent in

31 biodiversity conservation practices and modelling. The method outlined here can be used to

32 design robust surveillance systems for invasive species in a wider context, and to better tackle

33 uncertainty in protection of biodiversity and native species in a cost-effective manner. 
35 Invasive species can contribute to the extinction of native species, decrease species diversity, and

36 cause considerable ecological and economic loss ${ }^{1}$. Following initial introduction, invasive

37 species have a higher likelihood of being eradicated when detected early with a subsequent rapid

38 response ${ }^{2}$. While early detection requires effective surveillance to detect small numbers of

39 individuals ${ }^{3}$, often with limited resources.

40 Info-gap decision theory (IGDT) is a non-probabilistic theory that has been used in a range of

$41 \operatorname{areas}^{4}$. Such robust decision making methods are often desirable in ecological systems

42 characterized by Knightian uncertainty ${ }^{5}$, without considering the probability or frequency of

43 outcomes. IGDT offers an alternative of Wald's maximin to quantify the confidence in realising

44 specified aspirations but enable a balance between them as a robust-satisfying method ${ }^{6}$

45 Knightian uncertainty may exist in parameter estimates, regarded as parameter uncertainty.

46 IGDT has been used previously to manage invasive species (e.g. ${ }^{7,8,9}$ ), however, this project is

47 the first application of IGDT in determining the population threshold $(K)$ for detection of an

48 invasive species in order for it to be successfully eradicated. The population threshold ( $K$ )

49 represents a population number low enough to enable successful eradication without major

50 impact, but high enough that it can be detected without significant surveillance costs. Given that

51 the population threshold of a species is based on the risk tolerance of managers and the biology

52 of the species in question, it is difficult to provide actual values against this parameter. Despite

53 this, such management parameters are widely used (e.g. $\left.{ }^{10,11}\right)$. Because of the direct relation

54 between surveillance cost and population $\operatorname{size}^{12}$, these population threshold estimates could have

55 significant cost consequences if underestimated. 
56 Knightian uncertainty may also exist in uncertainty models and in system models (i.e. reward

57 function), known as structural or functional uncertainty (e.g. ${ }^{13}$ ). In the case of surveillance cost

58 and population threshold, how these two factors interact is likely to be different for each species

59 or for various surveillance period design, e.g. long-term spatial-dynamic models ${ }^{14}$ and static

60 optimisation methods over one-time period ${ }^{15}$. Using a different method for calculating

61 surveillance cost could potentially change the population threshold determined.

62 IGDT is a method that not only comprises uncertainty and economic deliberation, but also

63 quantitatively represents Knightian uncertainty where there is no objective measure of

64 probability $^{6}$. In our research, IGDT is applied to quantitatively represent the maximum Knightian

65 uncertainty against which robust decisions are generated. In this study, we use the potential

66 introduction of the Asian House Gecko, Hemidactylus frenatus (AHG), onto Barrow Island

67 (BWI), as a case study.

68 The Asian House Gecko as an excellent hitchhiker has expanded its range due to increased

69 vessel and goods movement ${ }^{16}$. AHG is of increasing concern for its potential effects on the

70 ecosystem. It is considered a high-risk species for incursion onto BWI. BWI was set aside as a

71 reserve by Western Australia legislature in 1910 due to its conservation value ${ }^{8}$. The Gorgon

72 Project commenced construction of a liquefied natural gas processing plant on BWI in $2009^{17}$. A

73 condition of the Gorgon Project approval was inclusion of a non-indigenous species surveillance

74 program that could detect an introduced species (with a statistical power of detection of 0.8 or

75 greater) if the species was present on the island due to Gorgon Project activities ${ }^{18}$. In order to

76 meet this ministerial requirement, a surveillance system model was developed ${ }^{19}$. This model is

77 primarily reliant on expert elicitation for input values, which may underestimate the uncertainty 
78 in the model parameters with lack of data and other potential and subtle independent

79 influences ${ }^{20}$, and result in exceeding budget limits.

80 This research was conducted to determine the robust population threshold required to trigger

81 detection of invasive species at a point that will reduce the likelihood of environmental damage.

82 Our study provides a new method for designing robust surveillance, contributing to efficient

83 application of limited resources and reducing financial and environmental costs.

\section{2. Methods}

\section{$85 \quad 2.1$ Data}

86 The Surveillance System for terrestrial vertebrates on BWI has undergone a large number of

87 reviews (e.g. ${ }^{8}$ ) since the commencement of the Gorgon Project. The reviews take into

88 consideration the operations being undertaken at the time. These include changes to the volume

89 of cargo, number of personnel travelling to BWI and the ports of origin. Our study is based on

90 revised input data from the most recent studies (e.g. ${ }^{21}$ ).

91 Our model considers six primary or secondary points of entry (Supplementary Table S1, Fig. 1).

92 In addition, three preferred habitat types were the focus of island surveillance for AHG

93 (Supplementary Table S2), as opposed to surveying the entire island. AHG are rarely found in

94 natural areas in their introduced range and prefer anthropologically modified areas, particularly

95 where there is artificial lighting that attracts insect prey ${ }^{22}$.

96 For the purpose of this model, BWI has been spatially separated into four zones (See Fig. 1 for

97 more details). AHG are excellent climbers, and are regularly found on walls, under buildings and

98 on ceilings ${ }^{23}$. For this reason, the area for surveillance in Zone 1 is focused on and around 
99 buildings and was calculated based on a three-dimensional searching area. The surveillance area

100 of each location is shown in Supplementary Table S3. There are six Surveillance System

101 Components (SSCs) $(i=1,2 \ldots, 6)$ used to detect the AHG on BWI (Supplementary Table S4).

102 The characters of these SSCs at different locations and zones are summarized in Supplementary

103 Table S5. The surveillance period designed for this model is one year since the probability of

104 AHG being detected changes due to seasonal elements within a year ${ }^{24}$.

1052.2 Model methods

106 Info-gap analysis requires three primary components, each of which builds on the last; a system

107 model, the performance requirement and the uncertainty model ${ }^{4}$. Following Jarrad et al. ${ }^{19}$ and

108 Whittle et al. ${ }^{24}$, the quantity of SSC $i$ to be used in each zone $j, N_{i}^{j}\left(N_{i}^{j} \geq 0\right)$, could be

109 calculated as equation (1):

110

$$
N_{i}^{j}=\frac{\log \left(\beta^{\left.\frac{1}{\left(1-\sigma_{i}^{j} F_{i}^{j}\right) C_{i}\left(1 / R^{j}\right)}\right)}\right.}{K \log \left(1-\sigma_{i}^{j} F_{i}^{j}\right)}\left(K \geq 0, \sigma_{i}^{j} \leq 1, F_{i}^{j} \leq 1, R^{j} \leq 1, C_{i}>0\right)
$$

111 Where $i$ is the type of SSC (Supplementary Table S4); $j$ is the risk zone; $R^{j}$ indicates the

112 probability of occupancy in risk zone $j ; \beta$ is the probability of a type 2 error (the probability of

113 falsely declaring the invasive species to be absent); $\sigma_{i}^{j}$ is the detection probability of SSC $i$ in

114 zone $j$ given invasive species present in the footprint; $F_{i}{ }^{j}$ is the sampling fraction covered by

115 one SSC unit of $i$ in zone $j$ (i.e. footprint/total target area); $K$ is the population threshold for

116 detection; $C_{i}$ is the cost per unit SSC $i$. 
117 Therefore, the system model in this research, i.e. the total surveillance cost used at all locations,

118 is $r=\sum_{i}\left\{\left(\sum_{L}\left(\sum_{j} \operatorname{ceil}\left(N_{i}^{j} \times \frac{A_{L}^{j}}{A_{t}^{j}}\right)\right)\right) \times C_{i}\right\}$, where $A_{L}^{j}$ is the area of zone $j$ at location $L, A_{t}^{j}$ is

119 the total area of zone $j$ (see Supplementary Section S1 for details).

120 The occupancy probabilities $\left(R^{j}\right)$ of AHG in Zone 0,1 and 2 during the early stage of invasion

121 is set to $0.1,0.8$ and 0.1 respectively, based on subject matter expert input. To reach Gorgon's

122 regulatory requirement of a specified detection power of $0.8, \beta$ is set to 0.2 . Wintle and

123 Burgman ${ }^{21}$ estimate the population threshold for AHG to be eight for the whole island

$124(\tilde{K}=8)$, based on subject matter expert elicitation and actual data from the eradication of AHG

125 from BWI in 2015, where seven AHGs were detected and eradicated. The value of other

126 parameter estimates have been given by independent vertebrate specialists either through expert

127 elicitation or surveying (Supplementary Table S5). The circumstances in which $K, \sigma, F, R$ are

128 uncertain will vary from study to study. For example, in circumstances where the footprint of all

129 SSCs for AHG can be measured precisely, there exists no uncertainty in the value of $F$.

130 Assume that the total surveillance cost is not able to exceed $r_{c}$, in other words, a budget limit of

$131 r_{c}$ is proposed. It is highly desirable that the total surveillance cost does not exceed the lower

132 value $r_{w}, r_{w} \leq r_{c}$.

133 We consider 40 different uncertain parameters: $K, \sigma_{i}^{j}, F_{i}^{j}$ and $R^{j}$ for $i=1, \ldots, 6$ and $j=0,1,2$.

134 We will refer generically to these 40 uncertain parameters with the vector $x=\left(x_{1}, \ldots, x_{40}\right)$ but

135 refer explicitly to $K, \sigma_{i}^{j}, F_{i}^{j}$ and $R^{j}$ for clarity or simplicity. We have initial parameter estimates

136 denoted by the known 40 -vector $\tilde{x}$. The only additional information available is that the 
137 parameters are all non-negative, and all parameters other than $K$ are bounded above by one. We

138 have no further information. For instance, probability distributions for uncertainty in these

139 parameters are lacking. In face of this severe uncertainty we will use fractional-error info-gap

140 model of uncertainty (equation (2)). Analogous to deviations around the mean in Bayesian

141 approach, info-gap uncertainty model (equation (2)) measures the fractional deviation between

142 the parameters and the estimates, but without probabilities assigned ${ }^{6}$.

$143 U(h)=\left\{x:\left|\frac{x_{n}-\tilde{x}_{n}}{\tilde{x}_{n}}\right| \leq h, x_{n} \geq 0, n=1: 40 ; \sigma_{i}^{j} \leq 1, F_{i}^{j} \leq 1, R^{j} \leq 1, i=1: 6, j=0: 2\right\}, h \geq 0$

144 Robustness can be mathematically explained as the greatest horizon of uncertainty up to which

145 the system model obeys the performance requirement, $r \leq r_{c}$. It measures the confidence in

146 realising the desired goals facing Knightian uncertainty. The robustness function is defined as

$147 \hat{h}\left(r_{c}\right)=\max \left\{h:\left(\max _{K, \sigma, F, R \in U(h)} r\right) \leq r_{c}\right\}$. Let $m(h)$ denote the inner maximum $(m(h)=\max r)$ and

$148 m(h)$ is the inverse function of $\hat{h}\left(r_{c}\right)$ (see Supplementary Section S1 for details).

149 Opportuneness is the least level of uncertainty that has to be tolerated to facilitate windfall

150 outcomes as wonderfully small as $r_{w}$. The opportuneness function in this model is

$151 \hat{\beta}\left(r_{w}\right)=\min \left\{h:\left(\min _{K, \sigma, F, R \in U(h)} r\right) \leq r_{w}\right\}$. Let $w(h)$ denote the inner minimum $(w(h)=\min r)$ and

$152 w(h)$ is the inverse function of $\hat{\beta}\left(r_{w}\right)$ (see Supplementary Section S2 for details).

153 All the exploratory analyses of robustness and opportuneness function have been conducted in 154 MATLAB R2018b ${ }^{25}$.

\section{3. Results}


156 The predicted surveillance cost based on the initial estimates of uncertainty parameters $\sigma, F, R$

157 decreases hyperbolically with an increasing AHG population threshold (Fig. 2A). The estimated

158 surveillance cost with the initial estimate for $K(\tilde{K}=8)$ and $\sigma_{i}^{j}, F_{i}^{j}, R^{j}$ is $\mathrm{AU} \$ 10,060$, which

159 seems to be a relatively small cost. However, these initial estimates are subjectively based on the

160 information experts had available at the time, and may not be necessarily accurate. To solve this

161 problem, the robustness is taken into consideration.

162 Fig. 2A illustrates that the surveillance cost is very large when $K$ is four or less, but the curve

163 flattens out when it is between six and twenty. This indicates that a $K$ value of between six and

164 twenty would be more economically sustainable. We therefore evaluated the robustness and

165 opportuneness using $K$ estimate values of 6, 8, 10 and 20.

166 Robustness curve when $K, \sigma, F, R$ are uncertain.

167 The step-wise continuity of these robustness curves is a result of the discrete values attainable by

$168 N$ and $K$, as expressed by the "ceil" function in Supplementary equation (S12). There are two

169 properties that should be noted, including zeroing and the trade-off property ${ }^{4}$.

170 The zeroing property affirms that predicted outcomes have zero robustness to uncertainty. Each

171 curve reaches the horizontal axis (for which robustness is zero) precisely when the required

172 performance equals the predicted value (Fig. 2B). For instance, consider the black curve,

173 calculated with an estimated surveillance cost of AU\$10,060, for which the robustness equals

174 zero (Fig. 2B). The trade-off property asserts that the robustness improves (increases) as the

175 performance requirement worsens (increases). This is reflected in the robustness curves of Fig. 
176 2B which increase monotonically: the robustness increases as the maximum allowed surveillance

177 cost increases; $r_{c}<r_{c}{ }^{\prime}$ implies $\hat{\alpha}\left(r_{c}\right) \leq \hat{\alpha}\left(r_{c}{ }^{\prime}\right)\left(\mathrm{p} 46,{ }^{4}\right)$.

178 Comparing four robustness curves in Fig. 2B, even though the minimized cost on curve ‘ $\tilde{K}=20$

179 ' is the lowest one, there is no guarantee that ' $\tilde{K}=20$ ' is the best choice for the underlying

180 inaccuracy in the parameter estimates. Curve ' $\tilde{K}=20$ ' is robust-preferred to the other three

181 curves at the same value of critical budget. For example, the largest error in the estimation of

182 parameters (i.e. robustness), $\pm 51 \%(0.51)$ would guarantee the total surveillance cost to be no

183 more than required AU $\$ 40,000$ when using ' $\tilde{K}=20$ ’.

184 Opportuneness curve when $K, \sigma, F, R$ are uncertain.

185 Robustness and opportuneness curves converge on the horizontal axis at the point reaching the

186 predicted surveillance cost, i.e. zeroing property (Fig. 2B). Zeroing for opportuneness means that

187 no uncertainty is needed in order to enable the expected outcome. Opportuneness decreases

188 along with the required surveillance cost. Opportuneness shows the immunity to windfall

189 success, thus the smaller it is the better. Opportuneness curve ' $\tilde{K}=20$ ' is lower than the other

190 three curves (Fig. 2B) and is thus preferred. We see that robustness and opportuneness are

191 synchronous in this example: Any change in $\tilde{K}$ that improves robustness, also improves

192 opportuneness.

\section{4. Discussion}

194 Ecological systems, and the species within, are highly complex and variable. This complexity

195 makes it difficult for model input parameter values to be derived from empirical data or for

196 uncertainty in these parameters to be parameterized probabilistically. This results in Knightian 
uncertainty underlying ecological analysis and consequently may skew policy outcomes. For

198 example, in our study, in a scenario in which the performance requirement is no more than a

199 critical budget value of AU\$40,000 (Fig. 2B), if any of the parameter estimates (when $\tilde{K}$ is

200 eight) err by more than $\pm 37 \%$, the total surveillance cost will be considerably over AU $\$ 40,000$.

201 To avoid the burden of excessive surveillance costs, it is critical to have a robust approach to

202 prevalent uncertainty, such as that outlined in this research.

203 Our research shows that the estimated cost for surveillance for a very small population threshold

204 (less than four) of AHGs is large (Fig. 2A). This is due to the fact that low population densities

205 reduce the probability of detection success, consequently for detection to occur, more resources

206 will be required ${ }^{12}$. An example of this is the annual trapping cost for gypsy moth in North

207 America, which Bogich et al. ${ }^{26}$ showed there was a linear function of trap density and total area,

208 with more traps required when the population size is small ${ }^{19}$. Counter to this, robustness

209 increases as size of the budget becomes larger (Fig. 2B).

210 Importantly, an increase in robustness allows for more mistakes in the parameter estimates to be

211 tolerated when making decisions, which is beneficial in pest management scenarios. One way to

212 improve robustness is by increasing the size of the budget (Fig. 2B). The other is by increasing

213 population threshold within the possible population threshold range, which simultaneously

214 improves opportuneness. The robustness gained with increasing the population threshold from

215 six to ten is similar to increasing the population threshold from ten to twenty at the same level of

216 required performance (Fig. 2B). Unrestrained increase in population threshold is not

217 recommended, as it could result in delay in detection, and subsequent failure to eradicate. How

218 big the population threshold is, is a matter of risk appetite. For robustness, 'bigger is better',

219 however, 'big is bad' for opportuneness ${ }^{4}$. A lower value associated with the opportuneness 
220 function means less uncertainty has to be tolerated, i.e. higher opportunity to seek lower

221 surveillance cost. In the case of AHG, the scenario in which ' $\tilde{K}=20$ ' (Fig. 2B) is not only

222 robust-dominant but is also opportuneness-dominant at the same level of required surveillance

223 cost, thus would be preferred by many decision makers. Davidovitch et al. ${ }^{8}$ pointed out that

224 decision makers are prone to being more risk averse rather than seeking windfalls.

225 Our methodology demonstrates how robustness/opportuneness curves may be applied to

226 determine if the size of the budget is 'sufficiently robust/opportune'. Determining a suitable

227 value of robustness/opportuneness is dependent upon the trade-offs deemed appropriate by

228 decision makers. If the decision-makers aim to reach ambitious goals (i.e. low critical

229 surveillance cost), they may need to compromise on the level of confidence in realizing them ${ }^{6}$.

230 Conversely, if decision-makers ideally have high confidence in realizing specified targets, then

231 the targets could be moderated by ambitiousness (i.e. they may need to improve the critical

232 surveillance cost). Such compromises in pest management requirements could be compensated

233 by achieving greater robustness against error in the parameter estimates.

234 The IGDT-based method explored in this research does not optimize (minimize) the cost spent

235 on surveillance but concentrates on identifying the robust population threshold against the

236 uncertainty existing in $\tilde{K}, \tilde{\sigma}, \tilde{F}, \tilde{R}$ for implementing eradication of a known pest, while

237 simultaneously satisfying a cost requirement. The method can easily be tailored to specific

238 requirements associated with varying degrees of uncertainty. As uncertainty parameters may not

239 be restricted to the parameters analysed in this research, more uncertainty parameters could be

240 included, e.g. the surveillance cost-effectiveness, population growth rate, discount rate that may

241 be considered in spatial-dynamic models analysis. On the other hand, if some of the parameters 
242 are based on reliable information and a smaller number of parameters are subjected to

243 uncertainty, the model could be simplified and potentially lead to more robust results. As Rout et

244 al. ${ }^{9}$ suggested, the same horizon of uncertainty $h$ can be applied to measure the uncertainty for

245 different parameters ( $K, \sigma, F, R$ in this research). The method gives decision makers freedom in

246 both what and how to tackle uncertainty to allow robust optimal decisions under varying

247 scenarios.

248 Early detection faces the challenge of reconciling limited budget with comprehensive sampling ${ }^{27}$.

249 Ecological uncertainty, distributional uncertainty and taxonomic uncertainty were recognised as

250 three basic underlying sources of uncertainty that may ultimately affect management decisions ${ }^{28}$.

251 This research offers a flexible application of IGDT that can be applied not only to the detection

252 of invasive species, but also incorporated into detection of rare and endangered species that are

253 inherently hard to find. This method may also be used directly in the robust surveillance design

254 in areas other than biodiversity protection, such as the grain industry and aquaculture. This

255 research could provide a guide to decision makers to determine other appropriate robust

256 thresholds regarding when and which strategy should be implemented for a variety of ecological

257 management purposes.

\section{Data availability}

259 Data available via the Dryad Digital Repository https://doi.org/10.5061/dryad.fxpnvx0rp

\section{References}

261 1. Ben-Haim, Y. Info-gap Decision Theory: Decisions Under Severe Uncertainty 2nd edn. $262 \quad$ (Academic Press, 2006). 
2. Ben-Haim, Y. \& Demertzis, M. Decision making in times of knightian uncertainty: An info-gap perspective. Economics 10, 1. https://doi.org/10.5018/economicsejournal.ja.2016-23 (2016).

3. Ben-Haim, Y. Management of invasive species: Info-gap perspectives. In Invasive species : risk assessment and management (eds Robinson, A., Walshe, T., Burgman, M. A., Nunn, M.) (Cambridge University Press, 2017).

4. Bogich, T. L., Liebhold, A. M. \& Shea, K. To Sample or Eradicate? A Cost Minimization Model for Monitoring and Managing an Invasive Species. Journal of Applied Ecology 45, 1134-1142. https://doi.org/10.1111/j.1365-2664.2008.01494.x (2008).

5. Commonwealth Government of Australia. Approval— Gorgon Gas Development (EPBC Reference: 2008/4178). Australian Capital Territory, Canberra (2009).

6. Davidovitch, L. et al. Info-gap theory and robust design of surveillance for invasive species: The case study of Barrow Island. Journal of Environmental Management 90, 2785-2793. https://doi.org/10.1016/j.jenvman.2009.03.011 (2009). 51. https://doi.org/10.4102/koedoe.v51i1.157 (2009).

280 8. Jarrad, F. C. et al. Improved design method for biosecurity surveillance and early detection of non-indigenous rats. New Zealand Journal of Ecology 35, 132-144 (2011). 300-302. https://doi.org/10.1046/j.1523-1739.1996.10010300.x (1996). 
11. Lodge, D. M. et al. Biological Invasions: Recommendations for U.S. Policy and Management. Ecological Applications 16, 2035-2054. https://doi.org/10.1890/10510761(2006)016[2035:BIRFUP]2.0.CO;2 (2006).

12. MathWorks. MATLAB R2018b. MathWorks, Natick, Massachusetts, USA (2018).

13. Mcdonald-madden, E., Peter, W. J. B. \& Possingham, H. P. Making Robust Decisions for Conservation with Restricted Money and Knowledge. The Journal of applied ecology 45, 1630-1638. https://doi.org/10.1111/j.1365-2664.2008.01553.x (2008).

14. McGinnis, S. M. \& Stebbins, R. C. A Field Guide to Western Reptiles and Amphibians 4th edn. (Houghton Mifflin Harcourt, 2018).

15. Mehta, S. V., Haight, R. G., Homans, F. R., Polasky, S., Venette, R. C. Optimal detection and control strategies for invasive species management. Ecological Economics 61, 237245. https://doi.org/10.1016/j.ecolecon.2006.10.024 (2007).

16. Metlay, D. From Tin Roof to Torn Wet Blanket: Predicting and Observing Ground Water Movement at a Proposed Nuclear Waste Site. In Prediction: science, decision making, and the future of nature (eds Sarewitz, D. R., Byerly, R., Pielke, R. A.) (Island Press, 2000).

303

17. Molina, R., Horton, T., Trappe, J. \& Marcot, B. Addressing uncertainty: How to conserve and manage rare or little-known fungi. Fungal Ecology 4, 134-146. https://doi.org/10.1016/j.funeco.2010.06.003 (2011). space and time. Methods in Ecology and Evolution 7, 891-899. https://doi.org/10.1111/2041-210X.12564 (2016). 
19. Pitt, J. P. W. Modelling the spread of invasive species across heterogeneous landscapes. Degree of Doctor of Philosophy, Lincoln University (2008).

20. Rödder, D., Solé, M. \& Böhme, W. Predicting the potential distributions of two alien invasive Housegeckos (Gekkonidae: Hemidactylus frenatus, Hemidactylus mabouia). North-Western Journal of Zoology 4, 236-246 (2008).

21. Rout, T. M., Thompson, C. J. \& McCarthy, M. A. Robust decisions for declaring eradication of invasive species. Journal of Applied Ecology 46, 782-786. https://doi.org/10.1111/j.1365-2664.2009.01678.x (2009).

22. Scott, J. K. et al. Zero-tolerance biosecurity protects high-conservation-value island

23. Sharov, A. A. Bioeconomics of Managing the Spread of Exotic Pest Species with Barrier Zones. Risk Analysis 24, 879-892. https://doi.org/10.1111/j.0272-4332.2004.00486.x (2004).

24. Trebitz, A. S. et al. Early detection monitoring for aquatic non-indigenous species: Optimizing surveillance, incorporating advanced technologies, and identifying research needs. Journal of Environmental Management 202, 299-310. https://doi.org/10.1016/j.jenvman.2017.07.045 (2017).

25. Vanderduys, E. \& Kutt, A. Is the Asian house gecko, Hemidactylus frenatus, really a threat to Australia's biodiversity? Australian Journal of Zoology 60, 361-367. https://doi.org/10.1071/ZO12077 (2013).

26. Whittle, P., Jarrad, F., Edwards, K. \& Mengersen, K. Design of the quarantine surveillance for non-indigenous species of invertebrates on Barrow Island. Records of the 


$$
\text { 122x.83.2013.113-130 (2013). }
$$

27. Wintle, B. \& Burgman, M. Expert Elicitation for Barrow Island Surveillance System Revision, Project Report. Centre of Excellence for Biosecurity Risk Analysis (CEBRA), Scenario Optimization Approach. Ecological Economics 133, 86-98. https://doi.org/10.1016/j.ecolecon.2016.11.018 (2017).

\section{Acknowledgements}

339 The authors thank Yakov Ben-Haim for useful comments. Many thanks to Grey Coupland for

340 editing, helpful advice and discussions, Cameron McMains for Fig. 1 and Lvyiqi Jiang for help

341 on application of Matlab. Also thanks to Chevron Australia for providing data used in this

342 research. This research was supported by the Harry Butler Institute, Murdoch University and

343 Qingdao Agricultural University.

\section{Author contributions}

345 YL conceived the ideas and designed methodology with guidance from SM; YL collected and 346 analysed the data with guidance from SM, MT, DZ, PHW; YL led the writing of the manuscript.

347 All authors contributed critically to the drafts and gave final approval for publication.

\section{$348 \quad$ Additional information}

349 Competing interests 
350 The authors declare no competing interests.

\section{$351 \quad$ Figure legends}

352 Fig. 1 Quarantine invasion risk map of the Asian House Gecko on Barrow Island. Zone 1 is the

353 area with the highest occupancy probability, where the majority of the surveillance budget

354 should be spent. This zone consists of areas where high risk activities for incursion are

355 undertaken. These activities include the unloading of cargo (laydown areas), the receipt of planes

356 (airports) and marine vessels (marine ports). Zone 2 is the secondary introduction area (100 m

357 buffer area around Zone 1) and is considered the lower risk boundary for a species dispersing out

358 of Zone 1. Zone 0 is the buffer area at Material Offloading Facility (MOF) (i.e. X-Blocs area).

359 This is because the habitat at Zone 2 at the MOF is highly different to that at all other locations, 360 making the characters of Surveillance System Components (SSCs) in Zone 2 at the MOF (i.e. X-

361 Blocs) different from that in Zone 2 at other locations. Zone 3 is the remaining island area where

362 the AHG is less likely to establish prior to detection thus with no SSCs allocated

363

364 Fig. 2 (A) Relationship between population threshold ( $K$ ) of Asian House Gecko and estimated

365 surveillance cost based on the initial estimates of uncertainty parameters $\sigma, F, R$ (Drawn

366 according to Supplementary equation (S3)); (B) Robustness/opportuneness curves when

$367 K, \sigma, F, R$ are uncertain using the initial estimates $\tilde{\sigma}, \tilde{F}, \tilde{R}$ and $\tilde{K}=6,8,10,20$ respectively.

368 Solid lines are robustness curves and dashed lines are opportuneness curves (Online version in 369 colour) 
Figures

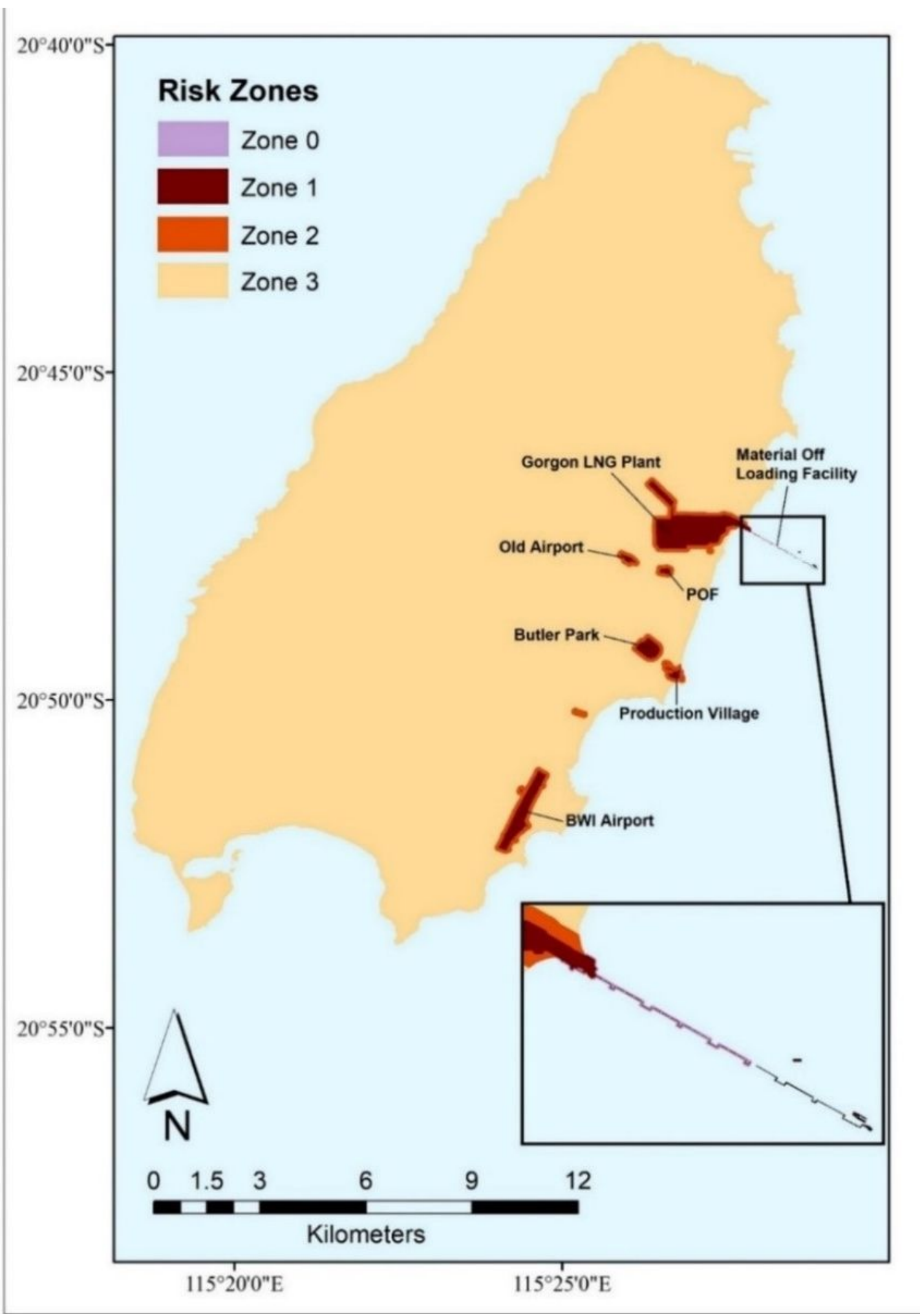

\section{Figure 1}

Quarantine invasion risk map of the Asian House Gecko on Barrow Island. Zone 1 is the area with the highest occupancy probability, where the majority of the surveillance budget should be spent. This zone consists of areas where high risk activities for incursion are undertaken. These activities include the 
unloading of cargo (laydown areas), the receipt of planes (airports) and marine vessels (marine ports). Zone 2 is the secondary introduction area (100 m buffer area around Zone 1) and is considered the lower risk boundary for a species dispersing out of Zone 1. Zone 0 is the buffer area at Material Offloading Facility (MOF) (i.e. X-Blocs area). This is because the habitat at Zone 2 at the MOF is highly different to that at all other locations, making the characters of Surveillance System Components (SSCs) in Zone 2 at the MOF (i.e. X Blocs) different from that in Zone 2 at other locations. Zone 3 is the remaining island area where the AHG is less likely to establish prior to detection thus with no SSCs allocated
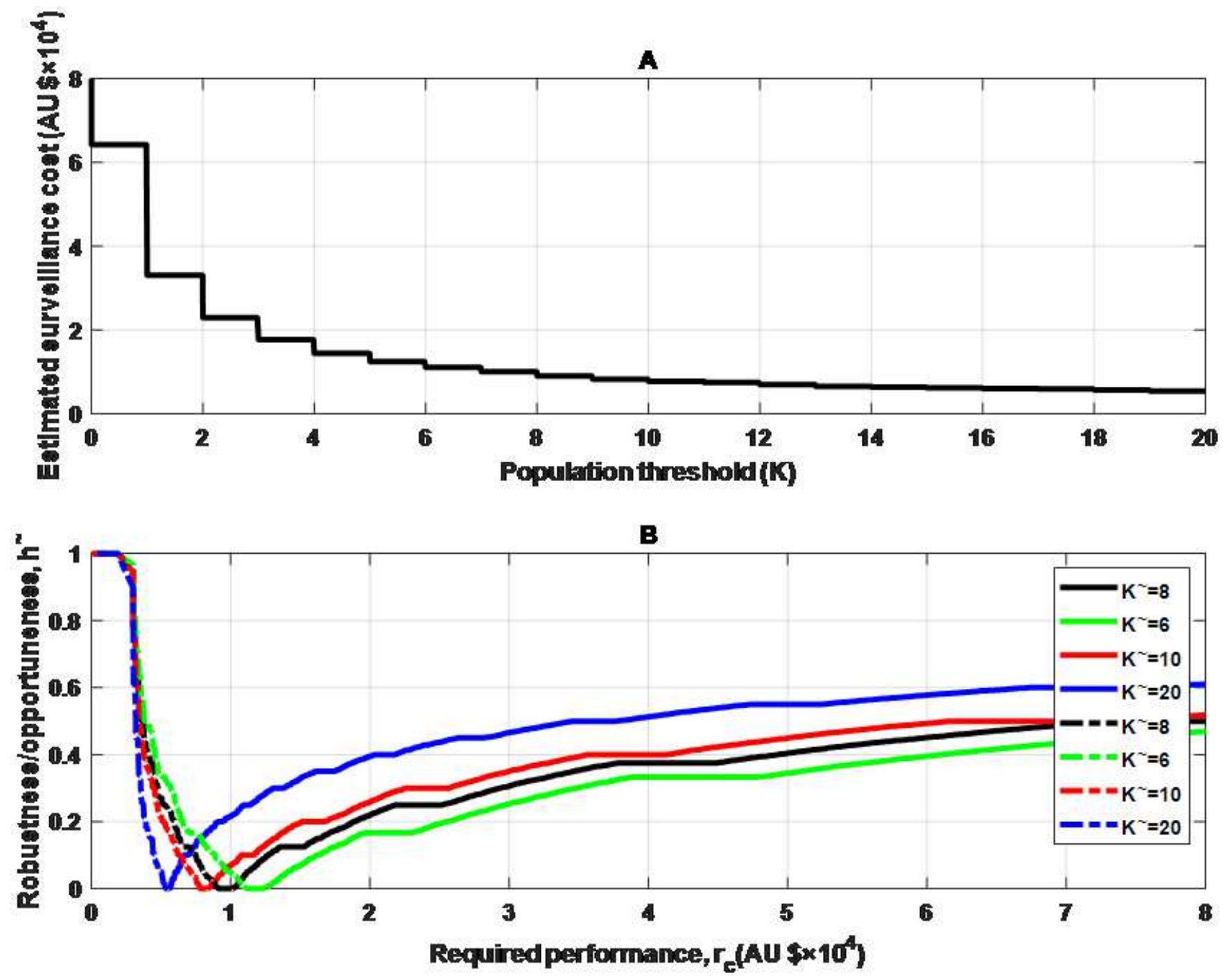

Figure 2

(A) Relationship between population threshold ( $K$ ) of Asian House Gecko and estimated surveillance cost based on the initial estimates of uncertainty parameters $\sigma, F, R$ (Drawn according to Supplementary equation (S3)); (B) Robustness/opportuneness curves when $\mathrm{K}, \sigma, \mathrm{F}, \mathrm{R}$ are uncertain using the initial estimates $\sigma, F, R$ and $K=6,8,10,20$ respectively. Solid lines are robustness curves and dashed lines are opportuneness curves (Online version in colour) 


\section{Supplementary Files}

This is a list of supplementary files associated with this preprint. Click to download.

- SupplementaryInformation.pdf 